\title{
Brittle and Ductile Behavior in Carbon Nanotubes
}

\author{
Marco Buongiorno Nardelli, B. I. Yakobson, and J. Bernholc \\ Department of Physics, North Carolina State University, Raleigh, North Carolina 27695-8202
}

(Received 27 August 1998)

\begin{abstract}
Large-scale molecular dynamics simulations were used to study the response of carbon nanotubes to a tensile load. Plastic or brittle behaviors can occur depending upon the external conditions and tube symmetry. All tubes are brittle at high strain and low temperature, while at low strain and high temperature armchair $(n, n)$ nanotubes can be completely or partially ductile. In zigzag $(n, 0)$ tubes ductile behavior is expected for tubes with $n<14$, while larger tubes are completely brittle. In both cases the curvature determines the mechanical response. [S0031-9007(98)07696-0]
\end{abstract}

PACS numbers: 61.48.+c, 61.72.Cc, 61.72.Lk, 62.20.Fe

The field of carbon nanotubes has seen an explosive growth in recent years due to the substantial promise of these quasi-1D structures for potential uses as highstrength, light-weight materials, super-strong fibers, novel nanometer-scale electronic and mechanical devices, catalysts, and energy storage media. Despite the potential impact that new composites based on carbon nanotubes could have in many areas of science and industry, a full characterization of their mechanical properties, and ultimately of their strength, is still lacking. Carbon nanotubes have already demonstrated exceptional mechanical properties: Their excellent flexibility during bending has been observed experimentally and studied theoretically [1-3]. Their high stiffness combines with resilience and the ability to buckle and collapse in a reversible manner: even largely distorted configurations (axially compressed, twisted) can be due to elastic deformations with virtually no atomic defects involved. [1,2,4,5] In this Letter we focus on the occurrence of mechanical failure in carbon nanotubes under a tensile load, which leads to the emergence of novel, unforeseen patterns in plasticity and breakage.

Because of its hexagonal symmetry, a graphite sheet (graphene), the basic constituent of carbon nanotubes, has three equivalent directions with respect to the application of an external planar tension. We call "longitudinal" the tension that is applied parallel to one of the $\mathrm{C}-\mathrm{C}$ bond directions, and "transverse" the one that is applied normal to it. Once the planar sheet is rolled into a nanotube, the case of the transverse tension corresponds to the application of tensile strain to an armchair tube, while the longitudinal case corresponds to the application of tensile strain to a zigzag tube. Our study, based on the extensive use of classical, tight-binding and $a b$ initio molecular dynamics simulations, shows that the different orientations of the carbon bonds with respect to the strain axis lead to completely different scenarios: ductile or brittle behaviors can be observed in nanotubes of different symmetry under the same external conditions. Furthermore, the behavior of nanotubes under large tensile strain strongly depends on their symmetry and diameter. Several modes of behavior are identified, and a full map of their ductile-vs-brittle behavior is presented.
Beyond a critical value of the tension, an armchair nanotube in "transverse" tension releases its excess strain via spontaneous formation of topological defects. A transverse tension finds a natural release in the rotation of the C-C bond perpendicular to it (the so-called StoneWales transformation [6]) which produces two pentagons and two heptagons coupled in pairs (5-7-7-5) [7]. The appearance of a (5-7-7-5) defect can be interpreted as the nucleation of a degenerate dislocation loop in the planar hexagonal network of the graphite sheet. The configuration of this primary dipole is a (5-7) core attached to an inverted (7-5) core. The (5-7) defect behaves thus as a single edge dislocation in the graphitic plane. Once nucleated the (5-7-7-5) dislocation loop can ease further relaxation by separating the two dislocation cores, which glide through successive Stone-Wales bond rotations $[7,8]$. This corresponds to a plastic flow of dislocations and gives rise to ductile behavior.

In contrast, in the case of a zigzag nanotube (longitudinal tension), the same $\mathrm{C}-\mathrm{C}$ bond will be parallel to the applied tension, which is already the minimum energy configuration for the strained bond. The formation of the Stone-Wales defect is then limited to rotation of the bonds oriented $120^{\circ}$ with respect to the tube axis. Our analysis shows that the formation energy of these defects is strongly dependent on curvature, i.e., on the diameter of the tube and gives rise to a wide variety of behaviors in the brittlevs-ductile map of stress response of carbon nanotubes.

Classical molecular dynamics simulations have been carried out for tubes of various geometries with diameters up to $13 \mathrm{~nm}$. The Tersoff-Brenner many-body potential was used to model the atomic interactions [9], but various key results were verified by tight binding and $a b$ initio simulations [10]. The real space multigrid approach was used in the $a b$ initio calculations [7,11], and the transferable carbon potential of Ho et al. [12] was employed in the tight-binding simulations.

We first consider the case of a "transverse" tension, i.e., an axial strain in an armchair tube. In the case of a molecularly perfect structure the limiting process for the yield and failure is the nucleation of the (5-7-7-5) defect in the ideal hexagonal network [7]. It has been shown 
that this process is energetically favored beyond a critical value of about 5\%-6\% tensile strain in both armchair tubes and the graphite sheet [7]. After the nucleation of such defect, the possible paths lead to either crack extension or separation and glide of the dislocation cores, through successive Stone-Wales rotations of different C-C bonds. We have investigated the energetics of further bond rotations in the presence of a preexisting (5-7-7-5) defect at various strains, mostly in the $(10,10)$ tube. The choice of this particular tube structure is due to the fact that $(10,10)$ tubes are the most abundant product in the laser ablation apparatus [13]. However, the results are qualitatively valid for all armchair tubes. Figure 1 shows the formation energies of the following: (i) An octagonal defect at the preexisting (5-7-7-5) defect as produced by a further Stone-Wales rotation of a strictly azimuthal bond; (ii) the initial stage of separation of the two dislocation cores (57) and (7-5), as produced by the rotation of the "shoulder" bond in the (5-7) core [8]. These results show that the separation of the dislocation cores is energetically favored over the formation of a larger open ring structure for strains up to $\approx 6 \%$. The calculated activation barriers are also lower for dislocation glide at strains lower than $\approx 6 \%$, and show a decrease with the decreasing formation energy [14]. One can then expect that for strains lower that $6 \%$ and at sufficiently high temperatures, the most commonly observed behavior will correspond to the separation and glide of the individual dislocation cores. For large strains and relatively low temperatures, on the other hand, one should expect the formation of larger ring structures.

The motion of dislocation edges in a strained structure is a well-known phenomenon in the theory of dislocations. Under uniform stress conditions in the limit of linear

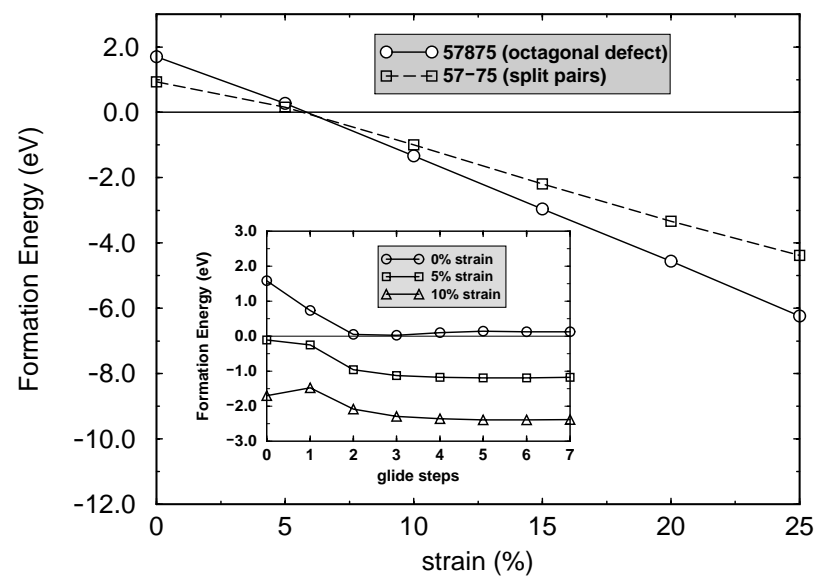

FIG. 1. Formation energy of an octagonal defect and of the initial step in the glide of the dislocation cores in a $(10,10)$ tube as a function of uniaxial strain. Inset: Formation energy for the glide of the dislocation core as a function of the glide step under three different strain conditions (each glide step corresponds to a bond rotation at the "shoulder" bond that separates the two cores of one lattice parameter). Note that the values of the dashed curve in the main panel correspond to the values for glide step $=1$ of the three curves in the inset. elasticity theory, the dislocation line is not fixed and the energy of the system can change if the dislocation moves. In particular, a glide of an edge dislocation via the successive rotation of the "shoulder" bond in the (5-7) core can reduce the total energy. Our results for the energetics of such a glide are summarized in the inset of Fig. 1. For all the strains considered, the initial energy gain is always smaller (more positive) in the first few gliding steps than in the large separation limit. This is the signature of a relatively long range attractive interaction between the two dislocation cores, which extends up to four gliding steps (four lattice parameters). For the glide of noninteracting dislocations, the activation barriers are significantly lower [15]. It is important to note that large strains are not needed in order to have a plastic flow of dislocations. In fact, it is clear from the inset in Fig. 1 that once the 5-77-5 dislocation cores are spatially separated, their motion is always energetically favored, and the tube will show a ductile behavior even for strains smaller than $5 \%$. Even though strain-induced dislocation loops are energetically favored to form at strain values $>5 \%$, one can expect that a certain number of such defects will be present in the asgrown tube $[7,16]$, thus making a ductile behavior possible.

For a better understanding of the kinetics of deformations and structural transformations associated with the ductile and brittle behaviors in armchair tubes, we studied the time evolution of a relatively long fragment $(3 \mathrm{~nm})$ of a $(10,10)$ tube under different strains and temperatures. The results of three different runs are summarized in Fig. 2. In Fig. 2a we show the initial formation of a (5-7-7-5) loop in a $10 \%$ stretched tube at $2000 \mathrm{~K}$ after 1.5 ns. This initial $10 \%$ strained configuration was then rescaled to a $3 \%$ strain through a sequence of coordinate rescaling and equilibration up to $3000 \mathrm{~K}$. The final 3\%strained configuration was then evolved for $2.5 \mathrm{~ns}$. Figure $2 \mathrm{~b}$ shows the final snapshot of the time evolution. The gliding of the individual dislocation cores is clear (shaded in the picture), demonstrating the expected plastic behavior at a relatively lower strain and high temperatures. In a second set of simulations, we further strained the initial single defect configuration up to $15 \%$, together with an equilibration to a lower temperature of $1300 \mathrm{~K}$. The system was then evolved for $\sim 1 \mathrm{~ns}$. Figure $2 \mathrm{c}$ shows the final snapshot of the time evolution. Under these different conditions, one readily observes the creation of octagonal defects and higher order rings. The initial defect acts as a nucleation center for other defects, whose extension leads to a brittle relaxation of the system.

The above simulations have shown that under the appropriate strain and temperature conditions armchair tubes display a ductile behavior through the plastic flow of (57) dislocations cores. It is well known that the introduction of topological defects can change the overall structure of nanotubes, e.g., the tube index or even the chirality. The remarkable feature of the glide of the (5-7) dislocation cores is related to the cylindrical symmetry of the carbon nanotube: the glide planes are spirals and the thermally 
a)

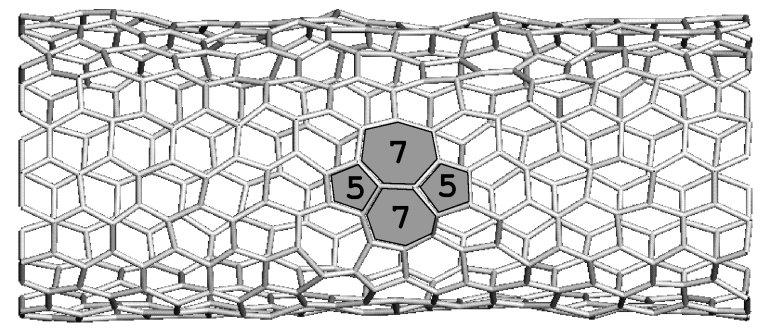

b)

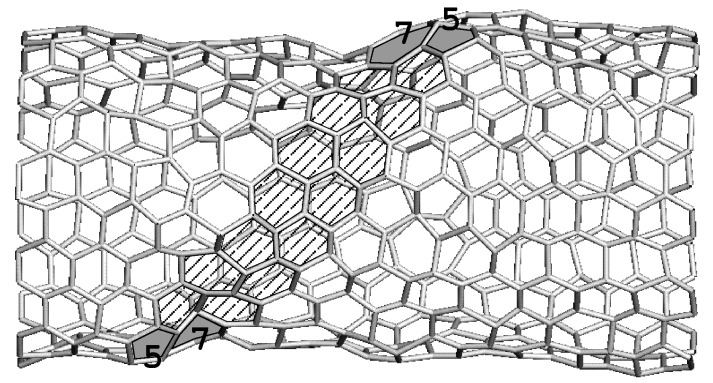

c)

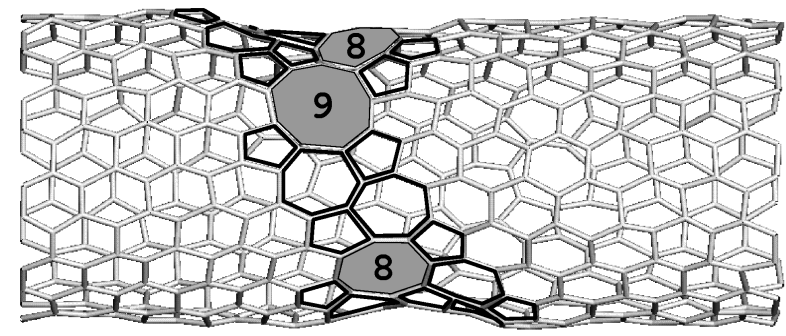

FIG. 2. Three snapshots of molecular dynamics simulations of a $(10,10)$ nanotube under axial tension. (a) Formation of a Stone-Wales defect at $T=2000 \mathrm{~K}$ and $10 \%$ strain; (b) plastic flow behavior after $\sim 2.5 \mathrm{~ns}$ at $T=3000 \mathrm{~K}$ and $3 \%$ strain. The shaded area indicates the migration path of the (5-7) edge dislocation; (c) nucleation of large open rings and initiation of the brittle relaxation after $\sim 1.0 \mathrm{~ns}$ at $T=1300 \mathrm{~K}$ and $15 \%$ strain. See text.

activated migration of the cores proceeds along these welldefined trajectories. Moreover, the 5-7 defect is the smallest defect that can change the tube index without drastically altering the local curvature of the nanotube [16-19]. When the dislocations sweep a sizable distance along the nanotube wall, they leave behind a tube segment changed according to the rules of dislocation theory [8]. In accord with these rules, the evolution of the $(n, n)$ tube will be: $(n, n) \rightarrow(n, n-1) \rightarrow(n, n-2) \ldots$ The tube thus abandons the armchair symmetry $(n, n)$ and, via the formation and splitting of new Stone-Wales defects, the relaxation leads it towards the zigzag symmetry $(n, 0)$. Correspondingly, this is manifested in a stepwise reduction of the diameter of the inner segment.

While in armchair tubes the plastic flow of dislocations and the consequent ductile behavior can be anticipated on the basis of the intrinsic geometry of the system, in a zigzag tube the consequences of the tensile strain are more subtle. Once the symmetry of the tube reaches the zigzag configuration, so that the tube is subject to a "longitudinal" tension, we find that its diameter plays a major role in determining the subsequent behavior. The results of static energetics calculations and molecular dynamics simulations for $(n, 0)$ tubes of various diameters $D$ at $10 \%$ strain are summarized in Fig. 3. Remarkably, the formation energy of the off-axis (5-7-7-5) defect (obtained via the rotation of the $\mathrm{C}-\mathrm{C}$ bond oriented $120^{\circ}$ with respect to tube axis) shows a crossover with respect to the diameter, and it is negative for $(n, 0)$ tubes with $n<14(D<1.1 \mathrm{~nm})$. Similarly, the formation energy of this defect in chiral tubes of the $(10, m)$ family (chosen as a particular example) is always negative, although it changes with the chiral angle $\chi$. This result implies that this geometrical transformation is still efficient in releasing the strain energy of the tube. The effect is clearly due to the variation in curvature, which in the small-diameter tubes makes the process energetically advantageous. Therefore, above a critical value of the curvature a plastic behavior is always possible and the tubes can be ductile. One can then expect that under low tensile load and high temperature conditions, a small diameter tube will undergo a series of geometrical transformations through a strain-induced plastic flow of dislocation cores. For example, using the rules of dislocation theory [8], the $(10,10)$ tube would undergo the following transformations: $(10,10) \rightarrow(10,9) \rightarrow(10,8) \ldots \rightarrow(10,0) \rightarrow[(9,1)$ or $(10,-1)] \ldots \rightarrow(9,0)$, etc.

In Fig. 4 we present a map of the ductile vs brittle behavior of a general $(n, m)$ carbon nanotube under an axial tensile load. There are four regions indicated by different shadings. The small hatched area near the origin is the region of complete ductile behavior, where the formation of (5-7-7-5) defects is always favored under sufficiently large strain. The plastic flow of single-core dislocations is thus possible under the appropriate strain and temperature conditions. In particular, plastic flow will transform the tube section between the dislocation cores along paths parallel to the axes of Fig. 4. During the

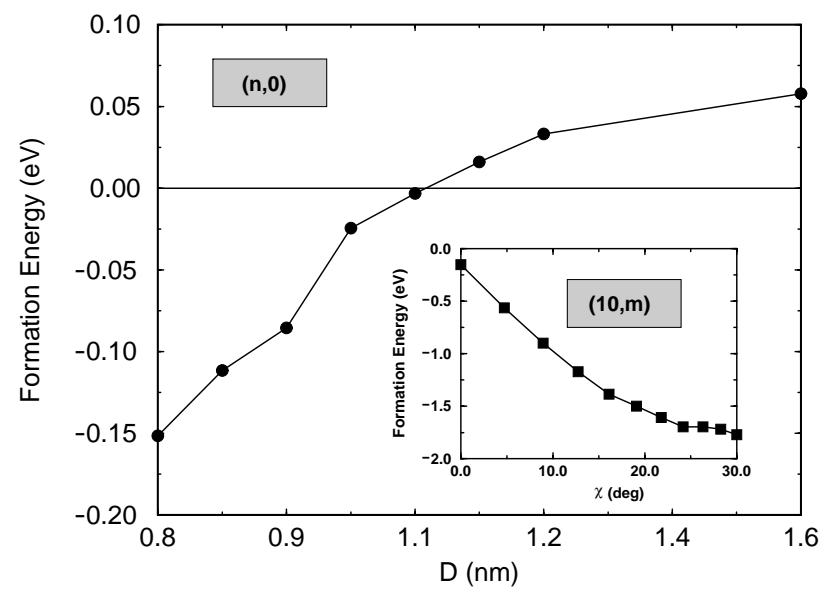

FIG. 3. Formation energy of the off-axis (5-7-7-5) defect in $(n, 0)$ tubes of various diameters. In $(n, 0)$ tubes, $D=$ $0.078 n \mathrm{~nm}$. Inset: Formation energy of the off-axis (5-7-7$5)$ defect in $(10, m)$ tubes of different chiralities. Note that in $(n, m)$ tubes the chiral angle $\chi=\arctan [\sqrt{3} m /(2 n+m)]$ is zero in zigzag tubes and $30^{\circ}$ in armchair tubes. All data refer to $10 \%$ strain. 


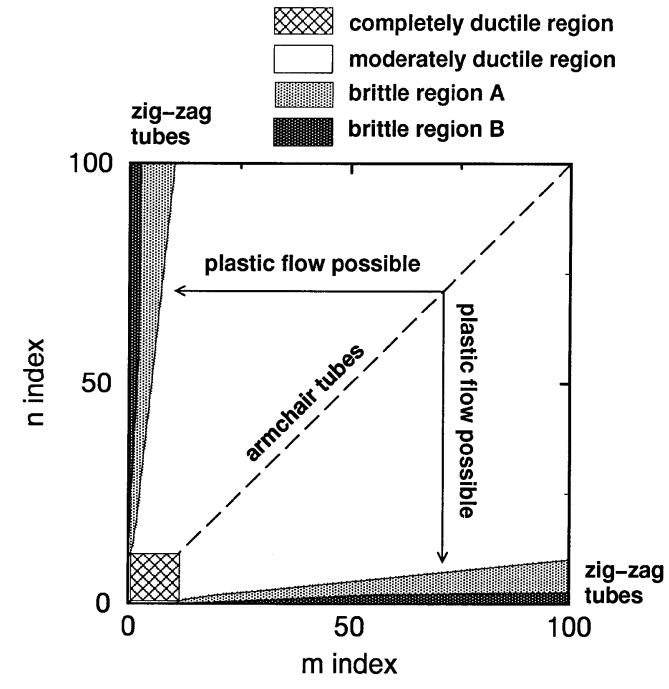

FIG. 4. Ductile-brittle domain map for carbon nanotubes with diameters up to $13 \mathrm{~nm}$. Different shaded areas correspond to different possible behaviors (see text).

transformations, the symmetry will oscillate between the armchair and the zigzag type. The same transformations will occur in the larger (white) moderately ductile region. Tubes with indices in this area are ductile, but the plastic behavior is limited by the brittle regions near the axes. The initial ductile behavior will eventually change the indices to the vicinity of the zigzag symmetry, where the calculations show that the tubes are completely brittle. In the "brittle region $A$ " the formation energy of the (5-7-7-5) defect is still negative, but other cracks are observed during molecular dynamics simulations. It will thus depend on the external conditions whether the plastic flow of dislocation is still possible, or a brittle fracture will occur. In the "brittle region $B$ " the (5-7-7-5) defect always has a positive formation energy and no plastic flow of dislocations is energetically favored to occur. This is the region of complete brittle behavior. Tubes that belong to this region will follow a brittle fracture path with formation of disordered cracks and large open rings under high tensile strain conditions.

In summary, several classes of behavior of carbon nanotubes under a tensile mechanical load have been identified through theoretical arguments and molecular dynamics simulations. After the nucleation of a first (5-7-7-5) defect in the hexagonal network, either brittle cleavage or plastic flow are possible, depending on tube symmetry, applied tension, and temperature. Under high strain and low temperature conditions, all tubes are brittle. If, on the contrary, external conditions favor plastic flow, such as low strain and high temperature, $(n, m)$ tubes with $n, m<14$ can be completely ductile, while larger tubes are moderately or completely brittle depending on their symmetry. The mechanism, energetics, and dynamics of the atomic rearrangements related to these regimes have been fully characterized and a map of the brittle vs ductile behavior has been presented.
We are pleased to acknowledge fruitful discussions with D. Orlikowsky and C. Roland.

[1] S. Iijima, C. Brabec, A. Maiti, and J. Bernholc, J. Chem. Phys. 104, 2089 (1996).

[2] B. I. Yakobson, C. J. Brabec, and J. Bernholc, Phys. Rev. Lett. 76, 2511 (1996).

[3] M. R. Falvo, G. J. Clary, R. M. Taylor II, V. Chi, F.P. Brooks, Jr., S. Washburn, and R. Superfine, Nature (London) 389, 582 (1997).

[4] J. Despres, E. Daguerre, and K. Lafdi, Carbon 33, 87 (1995).

[5] N. Chopra, L. Benedict, V. Crespi, M. L. Cohen, S. G. Louie, and A. Zettl, Nature (London) 377, 135 (1995); R. Ruoff and D. Lorents, Bull. Am. Phys. Soc. 40, 173 (1995).

[6] A. J. Stone and D. J. Wales, Chem. Phys. Lett. 128, 501 (1986).

[7] M. Buongiorno Nardelli, B. Yakobson, and J. Bernholc, Phys. Rev. B 57, R4277 (1998).

[8] B. I. Yakobson, Appl. Phys. Lett. 72, 918 (1998).

[9] J. Tersoff, Phys. Rev. Lett. 61, 2879 (1988); Phys. Rev. B 37, 6991 (1988); D. W. Brenner, Phys. Rev. B 42, 9458 (1990).

[10] In the $(5,5)$ tube the formation energy of the Stone-Wales defect at $0 \%$ and $10 \%$ strain is, respectively: 2.34 and $-1.77 \mathrm{eV}$ in ab initio [7], 1.84 and $-1.42 \mathrm{eV}$ in classical, 2.68 and -2.63 in tight-binding calculations. Note that all three sets of calculations predict that the formation of the Stones-Wales defect becomes energetically favored at about $5 \%$ strain. In zigzag $(10,0),(13,0)$, and $(16,0)$ tubes at $10 \%$ strain the formation energy of the same defect is, respectively: $-0.15,-0.02$, and $0.03 \mathrm{eV}$ in classical, and $-0.57,-0.20$, and 0.65 in tight-binding calculations. The crossover in the formation energy of the defect occurs in tubes of $\sim 1.1 \mathrm{~nm}$ diameter in both methods.

[11] E. L. Briggs, D. J. Sullivan, and J. Bernholc, Phys. Rev. B 52, R5471 (1995); E. L. Briggs, D. J. Sullivan, and J. Bernholc, Phys. Rev B 54, 14362 (1996).

[12] C. H. Xu, C.Z. Wang, C. T. Chan, and K. M. Ho, J. Phys. Condens. Matter 4, 3562 (1992).

[13] A. Thess, R. Lee, P. Nikolaev, H. Dai, P. Petit, J. Robert, C. Xu, Y.H. Lee, S. G. Kim, A. G. Rinzler, D. T. Colbert, G. Scuseria, D. Tomanek, J. B. Fisher, and R. E. Smalley, Science 273, 483 (1997).

[14] The activation barriers at $0 \%$ strain are 5.14 and $4.7 \mathrm{eV}$ for the formation energy of the octagonal defect and the initial separation of the (5-7) cores, respectively. These values are lowered to 3.1 and $3.4 \mathrm{eV}$ at $10 \%$ strain.

[15] The activations barrier for the initial separation of the two dislocation cores is $4.7 \mathrm{eV}$ in unstrained $(10,10)$ nanotubes, but it decreases to $3.0 \mathrm{eV}$ when the cores are separated by four lattice parameters.

[16] T. W. Ebbesen and T. Takada, Carbon 33, 973 (1995).

[17] B. I. Dunlap, Phys. Rev. B 46, 1933 (1992).

[18] Ph. Lambin, A. Fonseca, J.P. Vigneron, J. B. Nagy, and A. A. Lucas, Chem. Phys. Lett. 245, 85 (1995).

[19] L. Chico, V.H. Crespi, L. X. Benedict, S. G. Louie, and M. L. Cohen, Phys. Rev. Lett. 76, 971 (1996). 